\title{
Symmetry group classification for general Burgers' equation
}

\author{
M. Nadjafikhah ${ }^{\star}$, R. Bakhshandeh-Chamazkoti, \\ School of Mathematics, Iran University of Science and Technology, Narmak, Tehran 1684613114, Iran.
}

\begin{abstract}
The present paper solves the problem of the group classification of the general Burgers' equation $u_{t}=f(x, u) u_{x}^{2}+g(x, u) u_{x x}$, where $f$ and $g$ are arbitrary smooth functions of the variable $x$ and $u$, by using Lie method. The paper is one of the few applications of an algebraic approach to the problem of group classification: the method of preliminary group classification. A number of new interesting nonlinear invariant models which have nontrivial invariance algebras are obtained. The result of the work is a wide class of equations summarized in table form.
\end{abstract}

Key words: Infinitesimal generator, General Burgers' equation, Optimal system, Preliminarily group classification.

\section{Introduction}

It is well known that the symmetry group method plays an important role in the analysis of differential equations. The history of group classification methods goes back to Sophus Lie. The first paper on this subject is [1], where Lie proves that a linear two-dimensional second-order PDE may admit at most a three-parameter invariance group (apart from the trivial infinite parameter symmetry group, which is due to linearity). He computed the maximal invariance group of the one-dimensional heat conductivity equation and utilized this symmetry to construct its explicit solutions. Saying it the modern way, he performed symmetry reduction of the heat equation. Nowadays symmetry reduction is one of the most powerful tools for solving nonlinear partial differential equations (PDEs). Recently, there have been several generalizations of the classical Lie group method for symmetry reductions. Ovsiannikov [2] developed the method of partially invariant solutions. His approach is based on the concept of an equivalence group, which is a Lie transformation group acting in the extended space of independent variables, functions and their derivatives, and preserving the class of partial differential equations under study $[3,4,5]$.

The investigation of the exact solutions plays an important role in the study of nonlinear physical systems. A wealth of methods have been developed to find these exact physically significant solutions of a PDE though it is rather difficult. Some of the most important methods are the inverse scattering method [6], Darboux and Bäcklund transformations [7], Hirota bilinear method [7,8], Lie symmetry analysis [9,10,11], etc. The paper [12], based on the Lie group method, is investigated a very famous and important equation, which is the general Burgers' equation as the form

$$
u_{t}=a u_{x}^{2}+b u_{x x},
$$

where $u=u(x, t)$ is the unknown real function, $a, b \in \mathbb{R}$ and $a b \neq 0$. In the present paper, we consider the general Burgers' equation as the form

$$
u_{t}=f(x, u) u_{x}^{2}+g(x, u) u_{x x}
$$

\footnotetext{
* Corresponding author: Tel. +9821-73913426. Fax +9821-77240472.

Email addresses: m_nadjafikhah@iust.ac.ir (M. Nadjafikhah), r_bakhshandeh@iust.ac.ir (R. Bakhshandeh-Chamazkoti).
} 
where $u=u(x, t)$ is the unknown real function, $f$ and $g$ are arbitrary smooth functions of the variable $x$ and $u$. Eq. (1.2) represents the Burgers' equation combining both dissipative and nonlinear effects, therefore appears in a wide variety of physical applications. So it is important to lucubrate the exact explicit solutions and similarity reductions for this equation $[13,14]$. Here, we get the preliminary group classification of Eq. (1.2) by means of Lie point symmetry, and the constructed optimal systems of subalgebras. The knowledge of the optimal system of subalgebras gives the possibility of constructing the optimal system of solutions $[2,15,16]$ and permits the generation of new solutions starting from invariant or non-invariant solutions.

\section{Symmetry Methods}

Let a partial differential equation contains $p$ dependent variables and $q$ independent variables. The one-parameter Lie group of transformations

$$
\bar{x}_{i}=x_{i}+\epsilon \xi^{i}(x, u)+O\left(\epsilon^{2}\right) ; \quad \bar{u}_{\alpha}=u_{\alpha}+\epsilon \varphi^{\alpha}(x, u)+O\left(\epsilon^{2}\right),
$$

where $\xi^{i}=\left.\frac{\partial \bar{x}_{i}}{\partial \epsilon}\right|_{\epsilon=0}, i=1, \ldots, p$, and $\varphi^{\alpha}=\left.\frac{\partial \bar{u}_{\alpha}}{\partial \epsilon}\right|_{\epsilon=0}, \alpha=1, \ldots, q$, are given. The action of the Lie group can be recovered from that of its associated infinitesimal generators. we consider general vector field

$$
X=\sum_{i=1}^{p} \xi^{i}(x, u) \frac{\partial}{\partial x_{i}}+\sum_{\alpha=1}^{q} \varphi^{\alpha}(x, u) \frac{\partial}{\partial u^{\alpha}}
$$

on the space of independent and dependent variables. Therefore, the characteristic of the vector field $X$ given by (2.4) is the function

$$
Q^{\alpha}\left(x, u^{(1)}\right)=\varphi^{\alpha}(x, u)-\sum_{i=1}^{p} \xi^{i}(x, u) \frac{\partial u^{\alpha}}{\partial x_{i}}, \quad \alpha=1, \ldots, q
$$

The second prolongation of the infinitesimal operator

$$
X=\xi^{1}(x, t, u) \frac{\partial}{\partial x}+\xi^{2}(x, t, u) \frac{\partial}{\partial t}+\varphi(x, t, u) \frac{\partial}{\partial u} .
$$

obtained via the following prolongation formulas:

$$
X^{(2)}=X+\varphi^{x} \frac{\partial}{\partial u_{x}}+\varphi^{t} \frac{\partial}{\partial u_{t}}+\varphi^{x t} \frac{\partial}{\partial u_{x t}}+\varphi^{x x} \frac{\partial}{\partial u_{x x}},
$$

the coefficients are obtained by

$$
\varphi^{\iota}=D_{\iota} Q+\xi^{1} u_{x \iota}+\xi^{2} u_{t \iota}, \quad \varphi^{\iota \jmath}=D_{\iota}\left(D_{\jmath} Q\right)+\xi^{1} u_{x \iota \jmath}+\xi^{2} u_{t \iota \jmath},
$$

where $Q=\varphi-\xi^{1} u_{x}-\xi^{2} u_{t}$ is the characteristic of the vector field $X$ given by (2.5). For instance

$$
\begin{aligned}
\varphi^{x} & =D_{x} \varphi-u_{x} D_{x} \xi^{1}-u_{t} D_{x} \xi^{2}, \\
\varphi^{t} & =D_{t} \varphi-u_{x} D_{t} \xi^{1}-u_{t} D_{t} \xi^{2} \\
\varphi^{x x} & =D_{x} \varphi^{x}-u_{x x} D_{x} \xi^{1}-u_{x t} D_{x} \xi^{2} \\
\varphi^{x t} & =D_{t} \varphi^{x}-u_{x x} D_{t} \xi^{1}-u_{x t} D_{t} \xi^{2}
\end{aligned}
$$

where the operators $D_{x}$ and $D_{t}$ denote the total derivatives with respect to $x$ and $t$ :

$$
D_{x}=\frac{\partial}{\partial x}+u_{x} \frac{\partial}{\partial u}+u_{x x} \frac{\partial}{\partial u_{x}}+u_{x t} \frac{\partial}{\partial u_{t}}+\ldots, \quad D_{t}=\frac{\partial}{\partial t}+u_{t} \frac{\partial}{\partial u}+u_{t t} \frac{\partial}{\partial u_{t}}+u_{t x} \frac{\partial}{\partial u_{x}}+\ldots,
$$


By the theorem 6.5. in [17], $\left.X^{(2)}\left[u_{t}-f(x, u) u_{x}^{2}-g(x, u) u_{x x}\right]\right|_{(1.2)}=0$. Since

$$
X^{(2)}\left[u_{t}-f(x, u) u_{x}^{2}-g(x, u) u_{x x}\right]=\varphi^{t}-\left(f_{x} \xi^{1}+f_{u} \varphi\right) u_{x}^{2}-\left(g_{x} \xi^{1}+g_{u} \varphi\right) u_{x x}-2 f \varphi^{x} u_{x}-g \varphi^{x x},
$$

therefore we obtain the following determining function:

$$
\left.\left[\varphi^{t}-\left(f_{x} \xi^{1}+f_{u} \varphi\right) u_{x}^{2}-\left(g_{x} \xi^{1}+g_{u} \varphi\right) u_{x x}-2 f \varphi^{x} u_{x}-g \varphi^{x x}\right]\right|_{(1.2)}=0 .
$$

In the case of arbitrary $f(x, u)$ and $g(x, u)$ it follows that

$$
\xi^{1}=\varphi=\varphi^{x}=\varphi^{t}=\varphi^{x x}=0
$$

or

$$
\xi^{1}=\varphi=0, \quad \xi^{2}=C .
$$

Therefore, for arbitrary $f(x, u)$ and $g(x, u)$ Eq. (1.1) admits the one-dimensional Lie algebra $\mathfrak{g}_{1}$, with the basis

$$
X_{2}=\frac{\partial}{\partial t}
$$

$\mathfrak{g}_{1}$ is called the principle Lie algebra for Eq. (1.1). So, it is remained to specify the coefficient $f$ and $g$ such that Eq. (1.1) admits an extension of the principal algebra $\mathfrak{g}_{1}$. Usually, the group classification is obtained by inspecting the determining equation. But in our case the complete solution of the determining equation (2.12) is a wasteful venture. Therefore, we don't solve the determining equation but, instead we obtain a partial group classification of Eq. (1.1) via the so-called method of preliminary group classification. This method was applied when an equivalence group is generated by a finite-dimensional Lie algebra $\mathfrak{g} \mathscr{E}$. The essential part of the method is the classification of all nonsimilar subalgebras of $\mathfrak{g}_{\mathscr{E}}$. Actually, the application of the method is simple and effective when the classification is based on finite-dimensional equivalence algebra $\mathfrak{g}_{\mathscr{E}}$.

\section{Equivalence transformations}

An equivalence transformation is a nondegenerate change of the variables $t, x, u$ taking any equation of the form (1.1) into an equation of the same form, generally speaking, with different $f(x, u)$ and $g(x, u)$. The set of all equivalence transformations forms an equivalence group $\mathscr{E}$. We shall find a continuous subgroup $\mathscr{E}_{c}$ of it making use of the infinitesimal method.

We consider an operator of the group $\mathscr{E}_{C}$ in the form

$$
Y=\xi^{1}(x, t, u) \frac{\partial}{\partial x}+\xi^{2}(x, t, u) \frac{\partial}{\partial t}+\varphi(x, t, u) \frac{\partial}{\partial u}+\mu(x, t, u, f, g) \frac{\partial}{\partial f}+\nu(x, t, u, f, g) \frac{\partial}{\partial g},
$$

from the invariance conditions of Eq. (1.1) written as the system:

$$
\begin{aligned}
& u_{t}-f(x, u) u_{x}^{2}-g(x, u) u_{x x}=0 \\
& f_{t}=g_{t}=0
\end{aligned}
$$

where $u$ and $f, g$ are considered as differential variables: $u$ on the space $(x, t)$ and $f, g$ on the extended space $(x, t, u)$.

The invariance conditions of the system (3.17) are

$$
\begin{aligned}
& Y^{(2)}\left(u_{t}-f(x, u) u_{x}^{2}-g(x, u) u_{x x}\right)=0, \\
& Y^{(2)}\left(f_{t}\right)=Y^{(2)}\left(g_{t}\right)=0
\end{aligned}
$$


where $Y^{(2)}$ is the prolongation of the operator (3.16):

$$
Y^{(2)}=Y+\varphi^{x} \frac{\partial}{\partial u_{x}}+\varphi^{t} \frac{\partial}{\partial u_{t}}+\varphi^{x t} \frac{\partial}{\partial u_{x t}} \varphi^{x x} \frac{\partial}{\partial u_{x x}}+\mu^{t} \frac{\partial}{\partial f_{t}}+\nu^{t} \frac{\partial}{\partial g_{t}} .
$$

The coefficients $\varphi^{x}, \varphi^{t}, \varphi^{x t}, \varphi^{x x}, \varphi^{t t}$ are given in (2.7) and the other coefficients of (3.20) are obtained by applying the prolongation procedure to differential variables $f$ and $g$ with independent variables $(x, u)$. In view of $(3.18)$, we have

$$
\mu^{t}=\widetilde{D}_{t}(\mu)-f_{x} \widetilde{D}_{t}\left(\xi^{1}\right)-f_{u} \widetilde{D}_{t}(\varphi), \quad \nu^{t}=\widetilde{D}_{t}(\nu)-g_{x} \widetilde{D}_{t}\left(\xi^{1}\right)-g_{u} \widetilde{D}_{t}(\varphi)
$$

where $\widetilde{D}_{t}=\frac{\partial}{\partial t}$. So, we have the following prolongation formulas:

$$
\mu^{t}=\mu_{t}-f_{x} \xi_{t}^{1}-f_{u} \varphi_{t}, \quad \nu^{t}=\nu_{t}-g_{x} \xi_{t}^{1}-g_{u} \varphi_{t} .
$$

The invariance conditions (3.19) give rise to

$$
\mu^{t}=\nu^{t}=0,
$$

that is hold for every $f$ and $g$. Substituting (4.49) into (4.48), we obtain

$$
\mu_{t}=\nu_{t}=0, \quad \xi_{t}^{1}=\varphi_{t}=0 .
$$

Moreover with substituting (3.20) into (3.19) we obtain

$$
\varphi^{t}-2 f(x, u) u_{x} \varphi^{x}-g(x, u) \varphi^{x x}-\mu u_{x}^{2}-\nu u_{x x}-\nu=0 .
$$

Substituting (3.27), (3.28) and (3.29) into invariance condition (3.25), and introducing the relation $u_{t}=f(x, u) u_{x}^{2}+$ $g(x, u) u_{x x}$ to eliminate $u_{t}$ we are left with a polynomial equation involving the various derivatives of $u(x, t)$ whose coefficients are certain derivatives of $\xi^{1}, \xi^{2}, \varphi, \mu$, and $\nu$. We can equate the individual coefficients to zero, leading to the complete set of determining equations:

$$
\begin{aligned}
\xi^{1} & =\xi^{1}(x) \\
\xi^{2} & =\xi^{2}(t)=0 \\
\varphi_{u} & =\xi_{t}^{2} \\
\nu & =-g \xi_{t}^{2}+2 \xi_{x}^{1} \\
\mu & =-f \xi_{t}^{2}-f\left(\varphi_{u}-2 \xi_{x}^{1}\right)-g \varphi_{u u}
\end{aligned}
$$

so, we find that

$$
\begin{array}{rlrl}
\xi^{1}(x) & =a(x), & \xi^{2}(t)= & c_{1} t+c_{2}, \quad \varphi(x, u)=c_{1} u+b(x), \\
\mu & =-2 f\left(c_{1}-a(x)\right), \quad & \nu=-g\left(c_{1}-a^{\prime}(x)\right),
\end{array}
$$

with constants $c_{1}, c_{2}$ and two arbitrary functions $a(x)$ and $b(x)$ such that $b^{\prime \prime}(x)=c_{1}-a^{\prime}(x)$.

We summarize: The class of Eq. (1.2) has an infinite continuous group of equivalence transformations generated by the following infinitesimal operators:

$$
Y=a(x) \frac{\partial}{\partial x}+\left(c_{1} t+c_{2}\right) \frac{\partial}{\partial t}+\left(c_{1} u+b(x)\right) \frac{\partial}{\partial u}-2 f\left(c_{1}-a(x)\right) \frac{\partial}{\partial f}-g\left(c_{1}-a^{\prime}(x)\right) \frac{\partial}{\partial g},
$$

Therefore the symmetry algebra of the Burgers' equation (1.2) is spanned by the vector fields

$$
\begin{array}{ll}
Y_{1}=t \frac{\partial}{\partial t}+u \frac{\partial}{\partial u}-2 f \frac{\partial}{\partial f}-g \frac{\partial}{\partial g}, & Y_{2}=\frac{\partial}{\partial t}, \\
Y_{3}=a(x) \frac{\partial}{\partial x}+2 f a(x) \frac{\partial}{\partial f}+g a^{\prime}(x) \frac{\partial}{\partial g}, & Y_{4}=b(x) \frac{\partial}{\partial u} .
\end{array}
$$


Table 1

Commutation relations satisfied by infinitesimal generators in (4.35)

\begin{tabular}{llllll}
\hline$[]$, & $Y_{1}$ & $Y_{2}$ & $Y_{3}$ & $Y_{4}$ & $Y_{5}$ \\
\hline$Y_{1}$ & 0 & 0 & 0 & 0 & 0 \\
$Y_{2}$ & 0 & 0 & 0 & $Y_{2}$ & 0 \\
$Y_{3}$ & 0 & 0 & 0 & $Y_{3}$ & 0 \\
$Y_{4}$ & 0 & $-Y_{2}$ & $-Y_{3}$ & 0 & 0 \\
$Y_{5}$ & 0 & 0 & 0 & 0 & 0 \\
\hline
\end{tabular}

Table 2

Adjoint relations satisfied by infinitesimal generators in $(4.35)$

\begin{tabular}{llllll}
\hline$[]$, & $Y_{1}$ & $Y_{2}$ & $Y_{3}$ & $Y_{4}$ & $Y_{5}$ \\
\hline$Y_{1}$ & $Y_{1}$ & $Y_{2}$ & $Y_{3}$ & $Y_{4}$ & $Y_{5}$ \\
$Y_{2}$ & $Y_{1}$ & $Y_{2}$ & $Y_{3}$ & $Y_{4}-s Y_{2}$ & $Y_{5}$ \\
$Y_{3}$ & $Y_{1}$ & $Y_{2}$ & $Y_{3}$ & $Y_{4}-s Y_{3}$ & $Y_{5}$ \\
$Y_{4}$ & $Y_{1}$ & $e^{s} Y_{2}$ & $e^{s} Y_{3}$ & $Y_{4}$ & $Y_{5}$ \\
$Y_{5}$ & $Y_{1}$ & $Y_{2}$ & $Y_{3}$ & $Y_{4}$ & $Y_{5}$ \\
\hline
\end{tabular}

Moreover, in the group of equivalence transformations there are included also discrete transformations, i.e., reflections

$$
t \longrightarrow-t, \quad x \longrightarrow-x, \quad u \longrightarrow-u, \quad f \longrightarrow-f, \quad g \longrightarrow-g .
$$

\section{Preliminary group classification}

One can observe in many applications of group analysis that most of extensions of the principal Lie algebra admitted by the equation under consideration are taken from the equivalence algebra $\mathfrak{g}_{\mathscr{E}}$. We call these extensions $\mathscr{E}$-extensions of the principal Lie algebra. The classification of all nonequivalent equations (with respect to a given equivalence group $G_{\mathscr{E}}$, ) admitting $\mathscr{E}$-extensions of the principal Lie algebra is called a preliminary group classification. Here, $G_{\mathscr{E}}$ is not necessarily the largest equivalence group but, it can be any subgroup of the group of all equivalence transformations.

So, we can take any finite-dimensional subalgebra (desirable as large as possible) of an infinite-dimensional algebra with basis (3.33) and use it for a preliminary group classification. We select the subalgebra $\mathfrak{g}_{5}$ spanned on the following operators:

$$
\begin{aligned}
& Y_{1}=\frac{\partial}{\partial x}, \quad Y_{2}=\frac{\partial}{\partial t} \\
& Y_{4}=t \frac{\partial}{\partial t}+u \frac{\partial}{\partial u}-2 f \frac{\partial}{\partial f}-g \frac{\partial}{\partial g}, \quad Y_{5}=\frac{\partial}{\partial x}+2 f \frac{\partial}{\partial f}+g \frac{\partial}{\partial g} \text {. }
\end{aligned}
$$

The communication relations between these vector fields is given in Table 1. To each s-parameter subgroup there corresponds a family of group invariant solutions. So, in general, it is quite impossible to determine all possible group-invariant solutions of a PDE. In order to minimize this search, it is useful to construct the optimal system of solutions. It is well known that the problem of the construction of the optimal system of solutions is equivalent to that of the construction of the optimal system of subalgebras [2,15]. Here, we will deal with the construction of the optimal system of subalgebras of $\mathfrak{g}_{5}$.

Let $G$ be a Lie group, with $\mathfrak{g}$ its Lie algebra. Each element $T \in G$ yields inner automorphism $T_{a} \longrightarrow T T_{a} T^{-1}$ of the group $G$. Every automorphism of the group $G$ induces an automorphism of $\mathfrak{g}$. The set of all these automorphism is a Lie group called the adjoint group $G^{A}$. The Lie algebra of $G^{A}$ is the adjoint algebra of $\mathfrak{g}$, defined as follows. Let us have two infinitesimal generators $X, Y \in L$. The linear mapping $\operatorname{Ad} X(Y): Y \longrightarrow[X, Y]$ is an automorphism of 
$\mathfrak{g}$, called the inner derivation of $\mathfrak{g}$. The set of all inner derivations $\operatorname{ad} X(Y)(X, Y \in \mathfrak{g})$ together with the Lie bracket $[\operatorname{Ad} X, \operatorname{Ad} Y]=\operatorname{Ad}[X, Y]$ is a Lie algebra $\mathfrak{g}^{A}$ called the adjoint algebra of $\mathfrak{g}$. Clearly $\mathfrak{g}^{A}$ is the Lie algebra of $G^{A}$. Two subalgebras in $\mathfrak{g}$ are conjugate (or similar) if there is a transformation of $G^{A}$ which takes one subalgebra into the other. The collection of pairwise non-conjugate $s$-dimensional subalgebras is the optimal system of subalgebras of order $s$. The construction of the one-dimensional optimal system of subalgebras can be carried out by using a global matrix of the adjoint transformations as suggested by Ovsiannikov [2]. The latter problem, tends to determine a list (that is called an optimal system) of conjugacy inequivalent subalgebras with the property that any other subalgebra is equivalent to a unique member of the list under some element of the adjoint representation i.e. $\overline{\mathfrak{h}} \operatorname{Ad}(\mathrm{g}) \mathfrak{h}$ for some $\mathrm{g}$ of a considered Lie group. Thus we will deal with the construction of the optimal system of subalgebras of $\mathfrak{g}_{5}$.

The adjoint action is given by the Lie series

$$
\operatorname{Ad}\left(\exp \left(s Y_{i}\right)\right) Y_{j}=Y_{j}-s\left[Y_{i}, Y_{j}\right]+\frac{s^{2}}{2}\left[Y_{i},\left[Y_{i}, Y_{j}\right]\right]-\cdots,
$$

where $s$ is a parameter and $i, j=1, \cdots, 5$. The adjoint representations of $\mathfrak{g}_{5}$ is listed in Table 2 , it consists the separate adjoint actions of each element of $\mathfrak{g}_{5}$ on all other elements.

Theorem 4.1. An optimal system of one-dimensional Lie subalgebras of general Burgers' equation (1.2) is provided by those generated by

1) $Y^{1}=Y_{1}=\partial_{t}$,

2) $Y^{2}=Y_{2}=\partial_{x}$,

3) $Y^{3}=Y_{3}=\partial_{u}$,

4) $Y^{4}=Y_{4}=t \partial_{t}+u \partial_{u}-2 f \partial_{f}-g \partial_{g}$,

5) $Y^{5}=Y_{5}=\partial_{x}+2 f \partial_{f}+g \partial_{g}$,

6) $Y^{6}=Y_{1}+Y_{2}=\partial_{t}+\partial_{x}$,

7) $Y^{7}=-Y_{1}+Y_{2}=-\partial_{t}+\partial_{x}$,

8) $Y^{8}=Y_{1}+Y_{4}=(t+1) \partial_{t}+u \partial_{u}-2 f \partial_{f}-g \partial_{g}$

9) $Y^{9}=-Y_{1}+Y_{4}=(t-1) \partial_{t}+u \partial_{u}-2 f \partial_{f}-g \partial_{g}$,

10) $Y^{10}=Y_{1}+Y_{5}=\partial_{t}+\partial_{x}+2 f \partial_{f}+g \partial_{g}$,
11) $Y^{11}=-Y_{1}+Y_{5}=-\partial_{t}+\partial_{x}+2 f \partial_{f}+g \partial_{g}$,

12) $Y^{12}=Y_{4}+Y_{5}=t \partial_{t}+\partial_{x}+u \partial_{u}$,

13) $Y^{13}=-Y_{4}+Y_{5}=-t \partial_{t}+\partial_{x}-u \partial_{u}+4 f \partial_{f}+2 g \partial_{g}$,

14) $Y^{14}=Y_{1}+Y_{4}+Y_{5}=(t+1) \partial_{t}+\partial_{x}+u \partial_{u}$,

15) $Y^{15}=-Y_{1}+Y_{4}+Y_{5}=(t-1) \partial_{t}+\partial_{x}+u \partial_{u}$,

16) $Y^{16}=Y_{1}-Y_{4}+Y_{5}=(1-t) \partial_{t}-u \partial_{u}+2 f \partial_{f}+g \partial_{g}$,

17) $Y^{17}=-Y_{1}-Y_{4}+Y_{5}=-(1+t) \partial_{t}+\partial_{x}-u \partial_{u}+4 f \partial_{f}+2 g \partial_{g}$,

Proof. Let $\mathfrak{g}_{4}$ is the symmetry algebra of Eq. (1.2) with adjoint representation determined in Table 2 and

$$
Y=a_{1} Y_{1}+a_{2} Y_{2}+a_{3} Y_{3}+a_{4} Y_{4}+a_{5} Y_{5},
$$

is a nonzero vector field of $\mathfrak{g}$. We will simplify as many of the coefficients $a_{i} ; i=1, \ldots, 5$, as possible through proper adjoint applications on $Y$. We follow our aim in the below easy cases:

Case 1:

At first, assume that $a_{5} \neq 0$. Scaling $Y$ if necessary, we can assume that $a_{5}=1$ and so we get

$$
Y=a_{1} Y_{1}+a_{2} Y_{2}+a_{3} Y_{3}+a_{4} Y_{4}+Y_{5} .
$$

Using the table of adjoint (Table 2), if we act on $Y$ with $\operatorname{Ad}\left(\exp \left(a_{2} Y_{2}\right)\right)$, the coefficient of $Y_{2}$ can be vanished:

$$
Y^{\prime}=a_{1} Y_{1}+a_{3} Y_{3}+a_{4} Y_{4}+Y_{5}
$$

Then we apply $\operatorname{Ad}\left(\exp \left(a_{3} Y_{3}\right)\right)$ on $Y^{\prime}$ to cancel the coefficient of $Y_{3}$ :

$$
Y^{\prime \prime}=a_{1} Y_{1}+a_{4} Y_{4}+Y_{5} .
$$

Case 1a:

If $a_{1}, a_{4} \neq 0$ then we can make the coefficient of $Y_{1}$ and $Y_{4}$ either +1 or -1 . Thus any one-dimensional subalgebra 
generated by $Y$ with $a_{3}, a_{4} \neq 0$ is equivalent to one generated by $\pm Y_{1} \pm Y_{4}+Y_{5}$ which introduce parts 14), 15), 16) and 17) of the theorem.

Case 1b:

For $a_{1}=0, a_{4} \neq 0$ we can see that each one-dimensional subalgebra generated by $Y$ is equivalent to one generated by $\pm Y_{4}+Y_{5}$ which introduce parts 12) and 13) of the theorem.

Case 1c:

For $a_{1} \neq 0, a_{4}=0$ we can see that each one-dimensional subalgebra generated by $Y$ is equivalent to one generated by $\pm Y_{1}+Y_{5}$ which introduce parts 10) and 11) of the theorem.

Case 2:

The remaining one-dimensional subalgebras are spanned by vector fields of the form $Y$ with $a_{5}=0$. Case 2a:

If $a_{4} \neq 0$ then by scaling $Y$, we can assume that $a_{4}=1$. Now by the action of $\left.\operatorname{Ad}\left(\exp a_{2} Y_{2}\right)\right)$ on $Y$, we can cancel the coefficient of $Y_{2}$ :

$$
\bar{Y}=a_{1} Y_{1}+a_{3} Y_{3}+Y_{4} .
$$

Then by applying $\operatorname{Ad}\left(\exp \left(a_{3} Y_{3}\right)\right)$ on $\bar{Y}$ the coefficient of $Y_{3}$ can be vanished and we have

$$
\bar{Y}^{\prime}=a_{1} Y_{1}+Y_{4}
$$

The one-dimensional subalgebra generated by $Y$ is equivalent to one generated by $\pm Y_{1}+Y_{4}$ which introduce parts 8) and 9) of the theorem.

Case 2b:

Let $a_{4}=0$ then $Y$ is in the form

$$
\widehat{Y}=a_{1} Y_{1}+a_{2} Y_{2}+a_{3} Y_{3}
$$

Suppose that $a_{2} \neq 0$ then if necessary we can let it equal to 1 and we obtain

$$
\widehat{Y}^{\prime}=a_{1} Y_{1}+Y_{2}+a_{3} Y_{3} .
$$

By acting $\operatorname{Ad}\left(\exp \left(a_{3} Y_{3}\right)\right)$ on $\widehat{Y}^{\prime}$, it changed to $a_{1} Y_{1}+Y_{2}$ :

Case 2b-1:

Let $a_{1}$ be nonzero. In this case we can make the coefficient of $Y_{1}$ in $\widehat{Y}$ either +1 or -1 and find 6 ), 7) sections of the theorem.

Case 2b-2:

If $a_{1}$ is zero then $Y_{2}$ is remained. Hence this case suggests part 2).

Case 2c:

Finally if in the latter case $a_{2}$ be zero, then no further simplification is possible and then $Y$ is one of cases of (4.37). There is not any more possible case for studying and the proof is complete.

The coefficients $f, g$ of Eq. (1.2) depend on the variables $x, u$. Therefore, we take their optimal system's projections on the space $(x, u, f, g)$. The nonzero in $x$-axis or $u$-axis projections of (4.37) are
1) $Z^{1}=Y^{2}=Y^{6}=Y^{7}=\partial_{x}$,
4) $Z^{4}=Y^{5}=Y^{10}=Y^{11}=\partial_{x}+2 f \partial_{f}+g \partial_{g}$,
2) $Z^{2}=Y^{3}=\partial_{u}$,
5) $Z^{5}=Y^{12}=Y^{14}=Y^{15}=\partial_{x}+u \partial_{u}$,
3) $Z^{3}=Y^{4}=Y^{8}=Y^{9}=-Y^{16}=u \partial_{u}-2 f \partial_{f}-g \partial_{g}$,
6) $Z^{6}=Y^{13}=Y^{17}=\partial_{x}-u \partial_{u}+4 f \partial_{f}+2 g \partial_{g}$

Proposition 4.2. Let $\mathfrak{g}_{m}:=\left\langle Y_{1}, \ldots, Y_{m}\right\rangle$, be an m-dimensional algebra. Denote by $Y^{i}(i=1, \ldots, r, 0<r \leq$ $m, r \in \mathbb{N})$ an optimal system of one-dimensional subalgebras of $\mathfrak{g}_{m}$ and by $Z^{i}(i=1, \cdots, t, 0<t \leq r, t \in \mathbb{N})$ the projections of $Y^{i}$, i.e., $Z^{i}=\operatorname{pr}\left(Y^{i}\right)$. If equations

$$
f=\Phi(x, u), \quad g=\Psi(x, u),
$$


Table 3

The result of the classification

\begin{tabular}{lllll}
\hline$N$ & $Z$ & Invariant & Equation & Additional operator $X^{(2)}$ \\
\hline 1 & $Z^{1}$ & $u$ & $u_{t}=\Phi u_{x}^{2}+\Psi u_{x x}$ & $\partial_{x}, \partial_{t}+\partial_{x},-\partial_{t}+\partial_{x}$ \\
2 & $Z^{2}$ & $x$ & $u_{t}=\Phi u_{x}^{2}+\Psi u_{x x}$ & $\partial_{u}$ \\
3 & $Z^{3}$ & $x$ & $u_{t}=u^{2} \Phi u_{x}^{2}+u \Psi u_{x x}$ & $t \partial_{t}+u \partial_{u},(t+1) \partial_{t}+u \partial_{u},(t-1) \partial_{t}+u \partial_{u}$ \\
4 & $Z^{4}$ & $u$ & $u_{t}=e^{x^{2}} \Phi u_{x}^{2}+e^{x} \Psi u_{x x}$ & $\partial_{x}, \partial_{t}+\partial_{x},-\partial_{t}+\partial_{x}$ \\
5 & $Z^{5}$ & $\frac{u}{e^{x}}$ & $u_{t}=\Phi u_{x}^{2}+u \Psi u_{x x}$ & $t \partial_{t}+\partial_{x}+u \partial_{u},(t+1) \partial_{t}+\partial_{x}+u \partial_{u}$ \\
6 & $Z^{6}$ & $-\frac{1}{u}$ & $u_{t}=e^{x^{4}} \Phi u_{x}^{2}+e^{x^{2}} \Psi u_{x x}$ & $-t \partial_{t}+\partial_{x}-u \partial_{u},-(1+t) \partial_{t}+\partial_{x}-u \partial_{u}$ \\
\hline
\end{tabular}

are invariant with respect to the optimal system $Z^{i}$ then the equation

$$
u_{t}=\Phi(x, u) u_{x}^{2}+\Psi(x, u) u_{x x}
$$

admits the operators $X^{i}=$ projection of $Y^{i}$ on $(t, x, u)$.

Proposition 4.3. Let Eq. (4.48) and the equation

$$
u_{t}=\Phi^{\prime}(x, u) u_{x}^{2}+\Psi^{\prime}(x, u) u_{x x},
$$

be constructed according to Proposition 4.2. via optimal systems $Z^{i}$ and $Z^{i^{\prime}}$, respectively. If the subalgebras spanned on the optimal systems $Z^{i}$ and $Z^{i^{\prime}}$, respectively, are similar in $\mathfrak{g}_{m}$, then Eqs. (4.48) and (4.49) are equivalent with respect to the equivalence group $G_{m}$, generated by $\mathfrak{g}_{m}$.

Now we apply Proposition 4.2. and Proposition 4.3. to the optimal system (4.46) and obtain all nonequivalent Eq. (1.2) admitting $\mathscr{E}$-extensions of the principal Lie algebra $\mathfrak{g}$, by one dimension, i.e., equations of the form (1.2) such that they admit, together with the one basic operators (4.50) of $\mathfrak{g}$, also a second operator $X^{(2)}$. For every case, when this extension occurs, we indicate the corresponding coefficients $f, g$ and the additional operator $X^{(2)}$.

We perform the algorithm passing from operators $Z^{i}(i=1, \cdots, 6)$ to $f, g$ and $X^{(2)}$ via the following example. Let consider the vector field

$$
Z^{6}=\partial_{x}-u \partial_{u}+4 f \partial_{f}+2 g \partial_{g}
$$

then the characteristic equation corresponding to $Z^{6}$ is

$$
d x=\frac{d u}{-u}=\frac{d f}{4 f}=\frac{d g}{2 g},
$$

and can be taken in the form

$$
I_{1}=u e^{x}, \quad I_{2}=\frac{f}{e^{x^{4}}}, \quad I_{3}=\frac{g}{e^{x^{2}}} .
$$

From the invariance equations we can write

$$
I_{2}=\Phi\left(I_{1}\right), \quad I_{3}=\Psi\left(I_{1}\right),
$$

it follows that

$$
f=e^{x^{4}} \Phi(\lambda), \quad g=e^{x^{2}} \Phi(\lambda),
$$

where $\lambda=I_{1}$. 
From Proposition 4.2. applied to the operator $Z^{6}$ we obtain the additional operator $X^{(2)}$

$$
-t \partial_{t}+\partial_{x}-u \partial_{u}, \quad-(1+t) \partial_{t}+\partial_{x}-u \partial_{u}
$$

After similar calculations applied to all operators (4.46) we obtain the following result (Table 3) for the preliminary group classification of Eq. (1.2) admitting an extension $\mathfrak{g}_{3}$ of the principal Lie algebra $\mathfrak{g}_{1}$.

\section{Conclusion}

In this paper, following the classical Lie method, the preliminary group classification for the class of general Burgers' equation (1.2) and investigated the algebraic structure of the symmetry groups for this equation, is obtained. The classification is obtained by constructing an optimal system with the aid of Propositions 4.2. and 4.3.. The result of the work is summarized in Table 3. Of course it is also possible to obtain the corresponding reduced equations for all the cases in the classification reported in Table 3. We omitted these for brevity.

\section{References}

[1] S. Lie,: Arch. for Math. 6, 328 (1881).

[2] L. V. Ovsiannikov, Group Analysis of Differential Equations, Academic Press, New York, 1982.

[3] N. H. Ibragimov, M. Tottisi, and A. Valenti, Preliminary group classification of equations $u_{t t}=f\left(x, u_{x}\right) u_{x x}+g\left(x, u_{x}\right)$, J. Math. phys, 32, No. 11:2988-2995, 1991.

[4] Lina song, and Hongqing zhang, Preliminary group classification for the nonlinear wave equation $u_{t t}=f(x, u) u_{x x}+g(x, u)$, Nonlinear Analysis, (2008), doi:10.1016/j.na.2008.07.008.

[5] N. H. Ibragimov, M. Tottisi, and A. Valenti, Differential invariants of nonlinear equations $u_{t t}=f\left(x, u_{x}\right) u_{x x}+g\left(x, u_{x}\right)$, Communications in Nonlinear Science and Numerical Simulation 9 (2004) 6980.

[6] C.S. Gardner, J.M. Greene, M.D. Kruskal, R.M. Miura, Method for solving the Kortewegde Vries equation, Phys. Rev. Lett. 19 (1967) 10951097.

[7] Y.S. Li, Soliton and integrable systems, in: Advanced Series in Nonlinear Science, Shanghai Scientific and Technological Education Publishing House, Shang Hai, 1999 (in Chinese).

[8] R. Hirota, J. Satsuma, A variety of nonlinear network equations generated from the Bäcklund transformation for the Tota lattice, Suppl. Prog. Theor. Phys. 59 (1976) 64100.

[9] P.J. Olver, Applications of Lie group to Diferential Equations, in: Graduate Text Maths, vol. 107, Springer, New York, 1986.

[10] G.W. Bluman, S. Kumei, Symmetries and Differential Equations, Springer-Verlag, World Publishing Corp., 1989.

[11] B.J. Cantwell, Introduction to Symmetry Analysis, Cambridge University Press, 2002.

[12] Hanze Liu, Jibin Li and Quanxin Zhangb, Lie symmetry analysis and exact explicit solutions for general Burgers' equation, Journal of Computational and Applied Mathematics (2008), doi:10.1016/j.cam. 2008.06.009

[13] Mehdi Nadjafikhah, Lie symmetries of Burgers equation, Adv. appl. Clifford alg., DOI 10.1007/s00006-003-0000, 2008.

[14] Mehdi Nadjafikhah, Classification of similarity solutions for inviscid Burgers' equation, Accepted for Adv. appl. Clifford alg., DOI 10.1007/s00006 - 003-0000.

[15] M.L. Gandarias, M. Torrisi, A. Valenti, Symmetry classification and optimal systems of a non-linear wave equation, Int. J. Nonlinear Mech. 39 (2004) 389398.

[16] G.H. Maluleke , D.P. Mason, Optimal system and group invariant solutions for a nonlinear wave equation, Communications in Nonlinear Science and Numerical Simulation 9 (2004) 93104.

[17] P.J. Olver, Equivalence, Invariants, and Symmetry, Cambridge University Press, Cambridge, (1995). 